\title{
Aplicação de retardadores de crescimento em híbridos de girassol ornamental
}

\author{
GISELEAPARECIDABONACIN ${ }^{1}$; TERESINHA DE JESUS DELÉO RODRIGUES ${ }^{1 ;}$ CLAUDIAFABRINO MACHADO MATTIUZ
}

\begin{abstract}
RESUMO
O cultivo do girassol ornamental tem-se destacado nos últimos anos, como flor de corte e envasado. Diversas práticas de manejo são utilizadas na produção de plantas ornamentais envasadas, dentre elas o uso de retardadores de crescimento. O objetivo da pesquisa foi verificar o efeito de três retardadores de crescimento sobre o desenvolvimento de híbridos de girassol ornamental ('Sunbeam', 'Sunbright' e 'Sunbright Supreme'). O experimento foi desenvolvido em casa de vegetação na Faculdade de Ciências Agrárias e VeterináriasUNESP, Jaboticabal ( SP) A semeadura foi realizada em vasos de $2 \mathrm{~L}$ preenchidos com substrato Plantmax ${ }^{\circledR}$. As plantas receberam adubação constituída de Osmocote ${ }^{\circledR}$, Kristalon Branco®, Kristalon Laranja ${ }^{\circledR}$ e nitrato de cálcio, retiram-se as gemas laterais manualmente, deixando-se apenas a gema florífera apical. As soluções de paclobutrazol (3 mg i.a./vaso no substrato), cloreto de chlormequat ( $0,4 \mathrm{~g}$ i.a./vaso, via foliar) e daminozide $(0,5$ g i.a./vaso, via foliar) foram aplicadas quando as plantas estavam com 5 ou 6 pares de folhas (25 dias após a semeadura) e altura média de $21,45 \mathrm{~cm}$. O delineamento experimental foi em blocos casualizados, em esquema fatorial composto por três híbridos e três retardadores e mais as testemunhas, com quatro repetições. Não houve interação significativa entre os fatores para nenhuma das características avaliadas (altura, número de folhas, diâmetro do capítulo e área foliar). A menor altura de planta foi obtida com a aplicação de cloreto de chlormequat, que só diferiu $(\mathrm{P}<0,05)$ da testemunha no híbrido Sunbright observaram-se a maior altura, o menor capítulo e maior área foliar e os outros não diferiram entre si. Não houve diferenças significativas entre os híbridos nem entre os produtos quanto ao número de folhas. $\mathrm{O}$ daminozide induziu menor diâmetro do capítulo. A altura final atingida por todas as plantas que receberam os retardadores não foi suficiente para fins de comercialização em vaso.
\end{abstract}

Palavras-chaves: paclobutrazol, cloreto de chlormequat, daminozide, Helianthus annuus, plantas envasadas.

\begin{abstract}
Application of growth retardants in hybrids of ornamental sunflower.

The cultivation of ornamental sunflower has been increased during the past few years, as cut flower and pot plants. Several management practices are used for the production of pot ornamental plants, and among them the use of growth retardants. The objective of this research was to evaluate the effect of three growth retardants on development of hybrids of ornamental sunflower ('Sunbeam', 'Sunbright' and 'Sunbright Supreme'). The experiment was conducted under greenhouse conditions at Faculdade de Ciências Agrárias e Veterinárias UNESP- Câmpus de Jaboticabal-SP. The plants were fertilized with Osmocote, White Kristalon, Orange Kristalon and calcium nitrate and were conducted by pinching lateral buds, leaving only the apical flower bud. The solutions of paclobutrazol ( $3 \mathrm{mg} / \mathrm{pot}$, in the substratum), chlormequat chloride $(0.4 \mathrm{~g} /$ pot, leaf application) and daminozide $(0.5 \mathrm{~g} / \mathrm{pot}$, leaf application) were applied when the plants presented 5-6 leaf pairs (25 days after sowing) and average height of $21.45 \mathrm{~cm}$. The experimental design was in complete blocks and factorial arrangement of three hybrids and three retardants plus the control, with four replications. There was no significant interaction between the factors for all the characters analyzed (plant height, leaf number, head diameter and leaf area). The lowest value of plant height was obtained with the application of chlormequat chloride. The hybrid 'Sunbright' presented the highest height, smallest head and highest leaf area. There were no significant differences among hybrids nor among products in relation to leaf number. Daminozide induced lower head diameter and was different $(\mathrm{P}<0.05)$ from control plants. The final height of all plants that received the growth retardants was not enough for marketing.
\end{abstract}

Key words: paclobutrazol, chlormequat chloride, daminozide, Helianthus annuus, pot plants.

\footnotetext{
${ }^{1}$ Departamento de Biologia Aplicada à Agropecuária, FCAV-UNESP, Via de Acesso Professor Paulo Donato Castellane, km 5, 14884-900 - Jaboticabal (SP). E-mail: bonacin@ fcav.unesp.br.
} 


\section{INTRODUÇÃO}

No Estado de São Paulo, o mercado de plantas ornamentais é uma realidade, tanto em termos de geração de empregos quanto em volume de vendas e, desse modo, a atividade atrai cada vez mais interessados (AGRIANUAL, 2001). Nesse mercado, uma das opções de cultivo seria o girassol ornamental como flor de corte e cultivado em vasos.

As envasadas floríferas destacam-se pelo florescimento e por agregarem valores como o fácil manuseio, transporte e reposição e aquelas compactas e com flores coloridas são muito apreciadas pelos consumidores.

O cultivo do girassol como planta ornamental vem se destacando atualmente por ser uma alternativa de oferta de produto de ciclo curto, cores contrastantes e de fácil identificação pelo consumidor. Dentre os diversos cultivares de girassol utilizados para fins ornamentais, a série "Sun" da empresa Sakata Seed Company destaca-se pelos híbridos sem pólen, com altura de caule de 1,5 m e alta qualidade para flor de corte (HUANG, 1995).

Diversas práticas de manejo são utilizadas na produção de plantas ornamentais envasadas, dentre elas destaca-se o uso de reguladores vegetais, principalmente de retardadores de crescimento, utilizados com a finalidade de produzir plantas mais compactas e de maior aceitação comercial. De acordo com TANIMOTO (1987), a inibição da síntese de giberelinas pelos retardadores de crescimento pode reduzir o alongamento do caule e a expansão foliar.

O tratamento de muitas plantas pode ser realizado via foliar ou solo, com produtos a seguir: daminozide; chlormequat; ancimidol; paclobutrazol; uniconazole e hidrazida maleica (MH-30), durante a fase de rápido crescimento vegetativo ou mesmo logo após o início do florescimento, quando estão no tamanho limite da altura das plantas (HERTWIG, 1977). Alguns reguladores são recomendados para crisântemo: Ancimidol, Chlormequat, Cloreto de clorofórmio, Daminozide, Paclobutrazol, Brometo de piproclanil (LARSON, 1985).

Diversos trabalhos relatam a aplicação de reguladores em espécies pertencentes à família Asteraceae: em crisântemo (MENHENNETT, 1984), zínia (PINTO, 2003), girassol comum(WAMPLE eCULVER, 1983; AURAS, 1997).

De acordo com MEJIAS e RUANO (1990b), com a utilização de reguladores, é possível alterar a forma, altura e aspecto geral das plantas, ocasionando também outros efeitos como maior ramificação e o aparecimento prematuro de flores. No caso do girassol ornamental, uma das recomendações, para o cultivo como envasada florífera, é o controle da altura das plantas com retardadores de crescimento (WHIPKER et al., 1998).

Considerando as poucas informações referentes ao cultivo do girassol como planta envasada, objetivouse avaliar a influência dos retardadores de crescimento paclobutrazol, cloreto de chlormequat e daminozide sobre três híbridos de girassol ornamental.

\section{MATERIAL E MÉTODOS}

O experimento foi desenvolvido em casa-devegetação na Faculdade de Ciências Agrárias e Veterinárias-UNESP, no município de Jaboticabal, SP (595 $\mathrm{m}$ de altitude, latitude de $21^{\circ} 15^{\prime} 22^{\prime}$ ' $\mathrm{S}$ e longitude $\left.48^{\circ} 18^{\prime} 58^{\prime \prime} \mathrm{W}\right)$.

As sementes de girassol ornamental Sunbright Supreme, Sunbeame 'Sunbright foram obtidas da empresa Sakata Seed Company, de Bragança Paulista, SP.

Realizou-se a semeadura direta com duas ou três sementes por vaso (capacidade de $2 \mathrm{~L} ; 13,5 \mathrm{~cm}$ de altura) preenchidos com o substrato Plantmax ${ }^{\circledR}$. Aplicaram-se 4,5g de Osmocote $\AA$ : $15-10-10$ por vaso, na superfície do substrato. Após a emergência efetuou-se o desbaste deixando-se uma planta por vaso. Os demais adubos utilizados foram:; Kristalon Branco®: 15-5-30 + 3 (óxido de $\mathrm{Mg}$ com 1,8\% de Mg) + micronutrientes; Kristalon Laranja ${ }^{\circledR}$ $6-12-36$ + 3 (óxido de $\mathrm{Mg}$ com 1,8\% de $\mathrm{Mg}$ ) + micronutrientes e nitrato de cálcio $(15,5 \%$ de nitrogênio total: $14,5 \%$ de $\mathrm{N}$ nítrico+ $1 \%$ de $\mathrm{N}$ amoniacal).

Durante a fase vegetativa os vasos foram irrigados uma vez por dia com solução de Kristalon Branco (80g/100L de água) + solução de nitrato de cálcio (80g/ $100 \mathrm{~L}$ de água). Durante a fase de florescimento foi fornecida solução de Kristalon Laranja (80g/100L de água) + solução de nitrato de cálcio $(80 \mathrm{~g} / 100 \mathrm{~L}$ de água). As soluções foram preparadas semanalmente e armazenadas em um reservatório de amianto, devidamente tampado e mantido na casa-de-vegetação.

As plantas foram irrigadas manualmente, cada vaso recebendo quantidade de água suficiente para manter o substrato úmido. Todas as gemas laterais foram retiradas manualmente, deixando-se apenas a gema florífera apical, para que fosse produzida uma única inflorescência por planta.

Foram utilizados os seguintes retardadores de crescimento: paclobutrazol, cloreto de chlormequat e daminozide. As soluções de paclobutrazol (3 mg i.a./vaso, no substrato), cloreto de chlormequat ( $0,4 \mathrm{~g}$ i.a./vaso, via foliar) e daminozide $(0,5 \mathrm{~g}$ i.a./vaso, via foliar) foram aplicadas quando a planta estava com 5 ou 6 pares de folhas (25 dias após a semeadura) e altura média de 21,45 $\mathrm{cm}$. A quantidade de solução de daminozide e cloreto de chlormequat pulverizadas por planta foi de $200 \mathrm{~mL}$ e de solução de paclobutrazol colocada por vaso foi de $48 \mathrm{~mL}$.

$\mathrm{O}$ delineamento experimental foi em blocos casualizados, segundo esquema fatorial composto por três híbridos e três reguladores mais as testemunhas, com quatro repetições. Foram realizadas quatro avaliações; a primeira, antes da aplicação dos reguladores (com avaliação da altura das plantas, número de folhas e diâmetro do caule) e três avaliações com coleta de plantas, aos 07, 14 e 35 dias após a aplicação dos retardadores. Ao todo foram avaliadas 144 plantas.

Nas três avaliações com coleta de plantas, as seguintes variáveis foram determinadas: altura da planta (representada pela distância entre o nível do solo e o ponto 
de inserção do último par de folhas), número de folhas por planta, diâmetro do caule na região do corte, área foliar, massa seca do caule, pecíolos, lâminas, capítulos e raízes. Determinou-se a área foliar utilizando-se o sistema de análise digital Delta T Devices. Para a obtenção da massa seca, as lâminas foliares, pecíolos, caules e raízes foram colocados em sacos de papel e levados à estufa com circulação forçada de ar, a $\pm 70{ }^{\circ} \mathrm{C}$, até massa constante, com posterior pesagem em balança de precisão.

Os resultados foram verificados a partir da média de quatro plantas de cada tratamento, a cada avaliação. Esses dados foram submetidos à análise de variância (teste F) e as médias de tratamentos comparadas pelo teste de Tukey a $5 \%$ de probabilidade.

\section{RESULTADOS E DISCUSSÃO}

O padrão de crescimento para a altura foi crescente até a última avaliação e, na maioria das vezes, constatouse maiores valores em plantas não-tratadas (Figura 1). Em plantas tratadas, nota-se redução da altura naquelas que receberam a aplicação de daminozide, com médias de 123 cm, 107,88 cm e 105,5 cm para 'Sunbright', 'Sunbeam' e 'Sunbright Supreme', respectivamente (Figura 1 a, b e c). PINTO (2003) também verificou redução na altura de plantas de zínia com pulverização foliar única de daminozide $(2,5$; 3,75 e $\left.5,0 \mathrm{~g} . \mathrm{L}^{-1}\right)$

De acordo com a Sakata (2000), a altura média para os híbridos 'Sunbeam', 'Sunbright' e 'Sunbright Supreme', cultivados para corte é de 130 a 200 centímetros. Por ser material de corte desenvolvido em vasos, houve a redução de crescimento da planta, entretanto a redução foi maior ainda com a aplicação dos reguladores (Tabela 1).

Cabe ressaltar que, a altura final, atingida por todas as plantas que receberam os retardadores, não foi suficiente para fins de comercialização. Provavelmente, a aplicação única desses produtos não tenha sido suficiente. Sabe-se que os produtores de floríferas envasadas buscam plantas baixas, compactas e com boa proporcionalidade na relação planta-vaso (MOTOS e OLIVEIRA, 1998).

No presente trabalho, a aplicação dos retardadores foi realizada quando as plantas estavam com altura média de 21,45 cm. De acordo com CHRISTENSEN e FRIIS (1987) e ADRIANSEN (1985), a média de altura final para plantas envasadas é de 20,0 a 25,0 cm, portanto, em estudos futuros, sugere-se a aplicação dos retardadores antes das plantas atingirem a altura de $20 \mathrm{~cm}$.

Considerando as características avaliadas: altura, número de folhas, diâmetro do capítulo e área foliar observase que não houve interação significativa entre híbridos e reguladores (Tabela 1). Com relação aos híbridos, observamse diferenças significativas para as características altura, diâmetro do capítulo e a área foliar, em ‘Sunbeam'e ‘Sunbright Supreme' verificaram-se menor altura e menor área foliar quando comparados com 'Sunbright', em que se notou menor diâmetro do capítulo (Tabela 1).
Analisando-se conjuntamente os três híbridos para cada regulador, nota-se menor altura das plantas com a aplicação de cloreto de chlormequat, diferindo significativamente do controle (Tabela 1). HARTMANN et al. (1990) constataram que a aplicação via foliar de cloreto de chlormequat (2.000 ppm), em bico-de-papagaio (Euphorbia pulcherrima Willd.), produziu plantas com hastes de tamanho reduzido, com obtenção de uma copa compacta e homogênea. Já MENHENNETT (1984), trabalhando com crisântemo, verificou que o paclobutrazol foi efetivo no controle de altura quando comparado a plantas tratadas com daminozide e cloreto de chlormequat.

Com relação ao número de folhas, nos reguladores testados não houve diferenças significativas entre si; o mesmo resultado foi obtido em plantas de Zinnia elegans, por PINTO (2003), quando também verificou que uso de retardadores não implica redução no número de folhas na planta. Adicionalmente, a aplicação de cloreto de chlormequat em todos os híbridos de girassol ornamental provocou o aparecimento de manchas cloróticas nas folhas, comprometendo a qualidade das plantas. Para BARRET (1992), a pulverização de cloreto de chlormequat quase sempre causa fitotoxicidade, como manchas cloróticas devido a danos nos cloroplastos.

Menores valores de diâmetro de capítulo foram verificados com a aplicação de daminozide, diferindo significativamente apenas do controle, no entanto maior valor de área foliar foi obtido em plantas tratadas com esse regulador (Tabela 1 ).

Observaram-se, nos híbridos 'Sunbeam' e 'Sunbright Supreme', menores valores de massa seca de caule, pecíolos e lâminas, diferindo significativamente do 'Sunbright' (Tabela 2). Maior valor para massa seca de capítulos foi verificada para 'Sunbright Supreme', diferindo do 'Sunbright'.

Diversos autores verificaram redução na massa seca da planta com a aplicação de retardadores de crescimento (AURAS, 1997; COX e KEEVER, 1988; LATIMER, 1991), porém, nesse trabalho, tal fato foi observado apenas para a massa seca de caule e capítulo (Tabela 2).

\section{CONCLUSÕES}

1. No híbrido 'Sunbright', observam-se maior altura, maior área foliar e menor diâmetro de capítulo em relação aos dois outros híbridos.

2. Com a aplicação dos retardadores houve redução da massa seca de caule e de capítulo nas plantas.

4. A aplicação dos retardadores de crescimento quando as plantas estavam com a altura média de $21,45 \mathrm{~cm}$ não foi efetiva.

3. Os retardadores reduziram a altura final das plantas, entretanto não foram suficientes para atender às exigências de mercado para plantas envasadas. 


\section{AGRADECIMENTOS}

As autoras agradecem ao Departarmento de Vendas de Flores da empresa Sakata Seed Sudamérica, pela doação do material, e à Assistente Técnica de Desenvolvimento Márcia Guimarães Kobori, pelas sugestões.

\section{REFERÊNCIAS}

ADRIANSEN, E. Height control of Beloperon guttata by paclobutrazol. Acta Horticulturae, Wageningen, v.167, 1985.

AGRIANUAL 2001: anuário da agricultura brasileira. São Paulo: FNP Consultoria e Comércio, 2001. p. 337-340.

AURAS, A.E. Efeito do paclobutrazol sobre a morfologia e anatomia foliar, crescimento de parte aérea, distribuição de biomassa e trocas gasosas em girassol. 1997. 88f. Tese (Doutorado em Fisiologia Vegetal) - Universidade Federal de Viçosa, Viçosa, 1997.

BARRET, J.E. Mecanisms of action. In: OFA, Ohio Florists Association. Tips on the use of chemical growth regulators on floriculture crops. Ohio, 1992. p.12-18.

COX, D.A; KEEVER, G.J. Paclobutrazol inhibits growth of zinnia and geranium. HortScience, Alexandria, v.23, n.6, p.1029-1030, 1988.

CHRISTENSEN, O.V.; FRIIS, K. Research and development of unknown pot plants. Acta Horticulturae, Wageningen, v.205, p.33-37, 1987.

HARTMANN, H.T.; KESTER, D.E; DAVIES, F.T. Plant propagation: principles and practices. 5.ed. Englewood Cliffs: Prentice Hall, 1990. 647p.

HERTWIG, K.V. Manual de herbicidas desfolhantes, dessecantes e fitorreguladores. São Paulo: Agrônomica Ceres, 1977.480p.

HUANG, M.C. New ornamental crops in Asia. Acta Horticulturae, Wageningen, v. 397, p.49, 1995.
LARSON, R.A. Growth regulators in floriculture. Horticultural Reviews, New York, v.7, p.399-481, 1985.

LATIMER, J.G. Growth retardants affect landscape performance on Zinnia, Impatiens and Marigold. HortScience, Alexandria, v.5, n.26, p.557-560, 1991.

MARTINEZ,P..F.; LOPEZ, D., RODRIGO, M.C.; CARAZO, N. Lottus ssp. Growth control for potted plant production. Acta Horticulturae, Wageningen, n. 541, p. 207-214, 2000.

MEJIAS, R.J. \& RUANO, M.C. Los reguladores de crescimento. Aplicaciones praticas. In:__. El cultivo industrial de plantas em maceta. Réus: Ediciones de Horticultura SL, 1990, p.149-155.

MENHENNETT, R. Comparison of a new triazole retardant paclobutrazol (PP333) with ancymidol, chlorphonium chloride, daminozide, and piproctanyl bromide, on stem extension and inflorescence development in Chrysanthemum morifolium Ramat. Scientia Horticulturae, Amsterdan, v.24, p. 349-358, 1984.

MOTOS, J.R.; OLIVEIRA, M.J.G. Produção de crisântemo em vaso. Holambra: Ed. Flortec, 1998. 34p.

PINTO, A.C.R. Efeitos de tamanho de vaso, sistema de condução e reguladores vegetais no desenvolvimento $\mathrm{e}$ nas qualidade de cultivares de zínia. 2003. 166f. Tese (Doutorado em Agronomia) - Faculdade de Ciências Agrárias e Veterinárias, Universidade Estadual Paulista, Jaboticabal, 2003.

TANIMOTO, E. Giberellin dependent root elongation in Lactuca sativa: Recovery from growth retardant-supressed elongation with thickening by low concentration of GA3. Plant Cell Physiology, Kyoto, v.28, p.963-973, 1987.

WAMPLE, R.L; CULVER, E.B. The influence of paclobutrazol, a new growth regulator on sunflowers. Journal of the American Society for Horticultural Science, Alexandria, v.108, p.122-125, 1983.

WHIPKER, B.; DASOJU, S; MCCALL, I. Guide to sucessful pot sunflower production. North Carolina: Cooperative Extension Service, 1998. 4p. (Horticulture Information Leaflet, 562). 

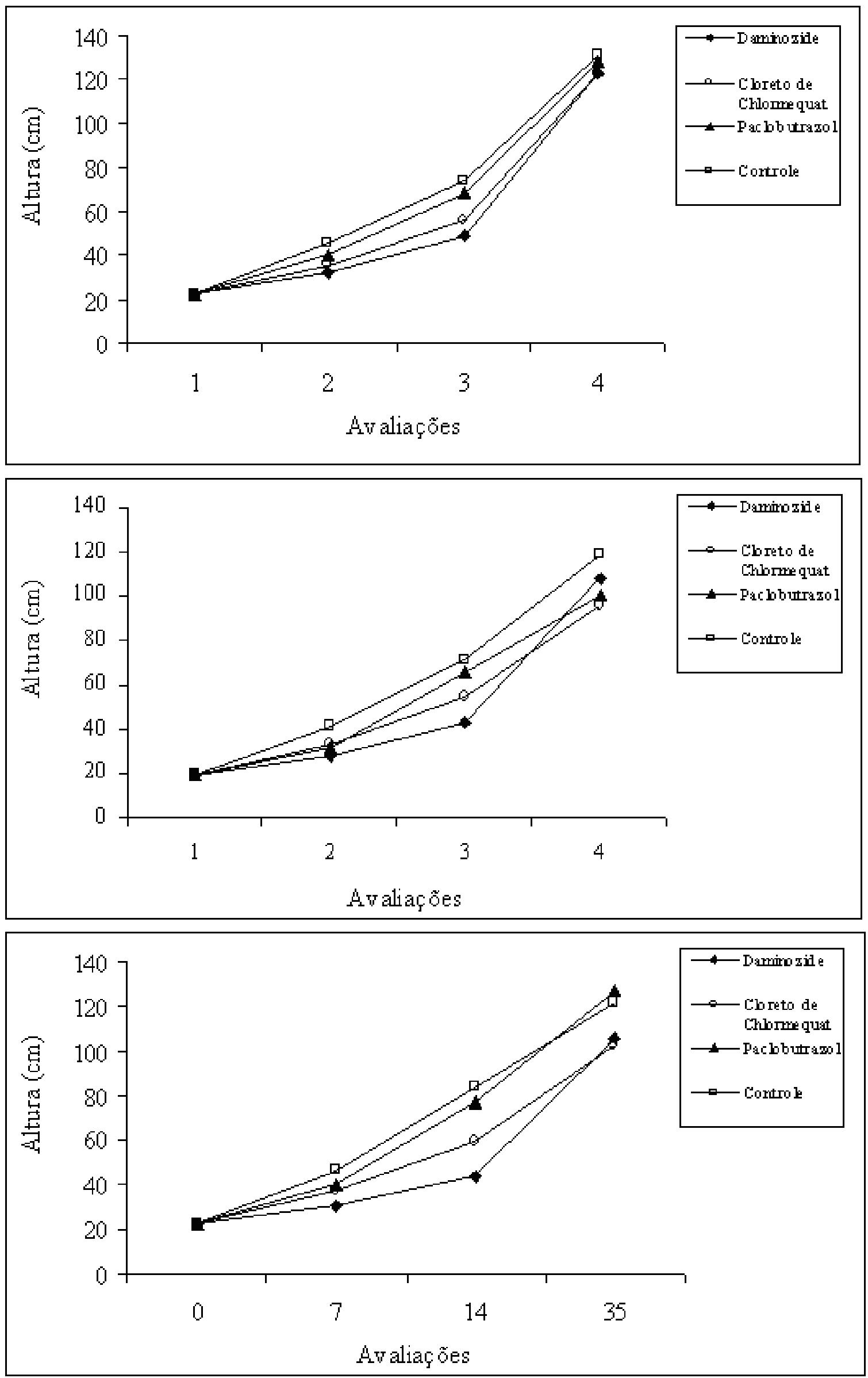

Figura 1. Crescimento em altura de plantas de girassol ornamental 'Sunbright' (a), 'Sunbeam' (b) e 'Sunbright Supreme' (c) tratadas ou não com Daminozide, Cloreto de Chlormequat (CCC) e Paclobutrazol. Jaboticabal (SP). 
Tabela 1. Altura, número de folhas, diâmetro do capítulo e área foliar, de três híbridos de girassol ornamental submetidos à aplicação de reguladores de crescimento na última avaliação

\begin{tabular}{|c|c|c|c|c|c|}
\hline & & Altura (cm) & $\begin{array}{l}\text { Número } \\
\text { de folhas }\end{array}$ & $\begin{array}{l}\text { Diâme tro do } \\
\text { capítulo (cm) }\end{array}$ & $\begin{array}{l}\text { Area foliar } \\
\text { (dro2) }\end{array}$ \\
\hline \multirow{4}{*}{ Hibridos } & 'Sunbeam' & $105,54 \mathrm{~b}$ & $20,75 \mathrm{a}$ & $18,58 \mathrm{a}$ & $20,90 \mathrm{~b}$ \\
\hline & 'Surbright' & $126,34 \mathrm{a}$ & $20,94 \mathrm{a}$ & $16,41 \mathrm{~b}$ & 24,72 a \\
\hline & 'Surbright Supre me' & $113,88 \mathrm{~b}$ & $19,69 \mathrm{a}$ & $21,41 \mathrm{a}$ & $20,08 \mathrm{~b}$ \\
\hline & d.m.s. & 10,22 & 1,79 & 2,55 & 1,70 \\
\hline \multirow{5}{*}{ Reguladores } & Controle & $123,65 \mathrm{~A}$ & $19,42 \mathrm{~A}$ & $20,58 \mathrm{~A}$ & $21,28 \mathrm{AB}$ \\
\hline & Paclobutrazol & $118,44 \mathrm{AB}$ & $21,33 \mathrm{~A}$ & $18,63 \mathrm{AB}$ & $22,14 \mathrm{AB}$ \\
\hline & Clore to de Chlormequat & $106,79 \mathrm{~B}$ & $19,58 \mathrm{~A}$ & $18,69 \mathrm{AB}$ & $20,77 \mathrm{~B}$ \\
\hline & Daminozide & $112,13 \mathrm{AB}$ & $21,50 \mathrm{~A}$ & $17,29 \mathrm{~B}$ & $23,42 \mathrm{~A}$ \\
\hline & d.m.s. & 13,01 & 2,28 & 3,24 & 2,17 \\
\hline \multicolumn{2}{|c|}{ Teste F (Hibridos-H) } & $12,66^{* *}$ & $1,71 \mathrm{~ns}$ & $11,68 * *$ & $25,50 * *$ \\
\hline \multicolumn{2}{|c|}{ Teste F (Reguladores-R) } & $4,68 * *$ & $2,55 \mathrm{~ns}$ & $3,48 *$ & $4,22 *$ \\
\hline \multicolumn{2}{|c|}{ Interação (H x R) } & $1,41 n s$ & $0,53 n s$ & $0,58 \mathrm{~ns}$ & $1,51 \mathrm{~ns}$ \\
\hline \multicolumn{2}{|l|}{$\mathrm{CV}(\%)$} & 10,21 & 10,09 & 15,61 & 8,95 \\
\hline
\end{tabular}

Médias seguidas por mesma letra maiúscula e minúscula na coluna não diferem entre si pelo teste de Tukey a 5\% de probabilidade. ns: não significativo; $* \mathrm{e}^{* *}$ respectivamente, significativo a $5 \%$ e $1 \%$ de probabilidade; d.m.s.: diferença mínima significativa.

Tabela 2. Massa seca de caule, pecíolos, lâminas, capítulos e raízes, de três híbridos de girassol ornamental submetidos à aplicação de reguladores de crescimento na última avaliação

\begin{tabular}{|c|c|c|c|c|c|c|}
\hline & & \multicolumn{5}{|c|}{ Masa Seca } \\
\hline & & Caule & Pecíolos & Lâminas & Capítulo & Raizes \\
\hline & & \multicolumn{5}{|c|}{ 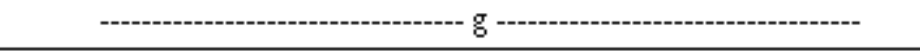 } \\
\hline \multirow{4}{*}{ Hibridos } & 'Surbeam' & $22,52 \mathrm{~b}$ & $3,76 \mathrm{~b}$ & $15,45 \mathrm{~b}$ & $16,78 \mathrm{ab}$ & $4,85 b$ \\
\hline & 'Sunbright' & 30,07 a & $5,39 \mathrm{a}$ & $19,03 \mathrm{a}$ & $13,01 \mathrm{~b}$ & $5,66 \mathrm{~b}$ \\
\hline & 'Surbright Supreme' & $23,45 \mathrm{~b}$ & $3,31 \mathrm{~b}$ & $14,82 \mathrm{~b}$ & $18,27 \mathrm{a}$ & $7,39 \mathrm{a}$ \\
\hline & d.m.s. & 2,93 & 0,62 & 1,92 & 4,34 & 1,18 \\
\hline \multirow{5}{*}{ Reguladores } & Controle & $29,85 \mathrm{~A}$ & $3,91 \mathrm{~A}$ & $15,82 \mathrm{~A}$ & $18,59 \mathrm{~A}$ & $5,92 \mathrm{~A}$ \\
\hline & Paclobutrazol & $26,47 \mathrm{AB}$ & $4,65 \mathrm{~A}$ & $17,43 \mathrm{~A}$ & $17,89 \mathrm{AB}$ & $6,61 \mathrm{~A}$ \\
\hline & Clore to de Chlormequat & $22,20 \mathrm{C}$ & $3,87 \mathrm{~A}$ & $15,90 \mathrm{~A}$ & $14,66 \mathrm{AB}$ & $5,61 \mathrm{~A}$ \\
\hline & Daminozide & $22,87 \mathrm{BC}$ & $4,19 \mathrm{~A}$ & $16,56 \mathrm{~A}$ & $12,94 \mathrm{~B}$ & $5,74 \mathrm{~A}$ \\
\hline & d.m.s. & 3,73 & 0,79 & 3,24 & 2,17 & 1,51 \\
\hline \multicolumn{2}{|c|}{ Teste F (Hibridos-H) } & $12,66^{* * *}$ & $1,71 \mathrm{~ns}$ & $11,68 * *$ & $25,50 * *$ & $14,48^{* * *}$ \\
\hline \multicolumn{2}{|c|}{ Teste F (Reguladores-R) } & $4,68 * *$ & $3,48 *$ & $2,55 \mathrm{~ns}$ & $4,22 *$ & $1,28 \mathrm{~ns}$ \\
\hline \multicolumn{2}{|c|}{ Interação (H x R) } & $1,41 \mathrm{~ns}$ & $0,53 \mathrm{~ns}$ & $0,58 \mathrm{~ns}$ & $1,51 \mathrm{~ns}$ & $2,41^{*}$ \\
\hline \multicolumn{2}{|l|}{$\mathrm{CV}(\%)$} & 10,21 & 10,09 & 15,61 & 8,95 & 22,83 \\
\hline
\end{tabular}

Médias seguidas por mesma letra maiúscula e minúscula na coluna não diferem entre si pelo teste de Tukey a $5 \%$ de probabilidade. ns: não significativo; $* \mathrm{e}^{* *}$ respectivamente, significativo a $5 \%$ e $1 \%$ de probabilidade; d.m.s.: diferença mínima significativa. 